\title{
Genotype-Guided Antiplatelet Therapy Versus Standard Therapy for Patients with Coronary Artery Disease: An Updated Systematic Review and Meta-Analysis
}

\author{
Borui Tang ${ }^{1}$, Xin Wang ${ }^{1}$, Xinrui Wang ${ }^{1,3}$, Lihong Liu ${ }^{1,2}$, Zhuo Ma ${ }^{1}$ \\ ${ }^{1}$ Department of Pharmacy, Beijing Chao-Yang Hospital, Capital Medical University, Beijing, China; ${ }^{2}$ Department of Pharmacy, \\ China-Japan Friendship Hospital, Beijing, China; ${ }^{3}$ Department of Pharmacy, Beijing Obstetrics and Gynecology Hospital, \\ Capital Medical University, Beijing, China.
}

Corresponding authors: Lihong Liu, Department of Pharmacy, China-Japan Friendship Hospital, Beijing, 100029, China; TEL: (+86)10 84205288; email: liulihong@bjcyh.com; Zhuo Ma, Department of Pharmacy, Beijing Chao-Yang Hospital, Capital Medical University, Beijing, 100020, China; TEL: (+86)10 85231077; email: mazhuo2013@163.com

Received, July 24, 2021; Revised, Dec 6, 2021; Accepted, December 22, 2021; PublishedJanuary 2, 2022

\begin{abstract}
Objective: Previous studies on the efficacy and safety of genotype-guided antiplatelet therapy in patients with coronary artery disease (CAD) or undergoing percutaneous coronary intervention (PCI) have been inconclusive. Aim: We conducted a meta-analysis to evaluate if the genotype-guided antiplatelet strategy is superior to the standard therapy in patients with CAD or undergoing PCI. Method: PubMed, Web of Science, Embase, and Cochrane Central Register of Controlled Trials databases were searched up to October $1^{\text {st }}, 2021$. Studies reporting efficacy and safety outcomes in the genotype-guided treatment and standard treatment groups were included. The two groups were statistically compared. Result: Eleven randomized controlled trials (RCTs) involving 11740 patients were included in this meta-analysis. Compared with the standard treatment group, the genotype-guided group had significant lower risks of all efficacy outcomes, including major adverse cardiovascular events (MACEs) (RR 0.60, 95\%, CI 0.44-0.82, $\mathrm{P}=0.001$ ), all-cause death (RR 0.70, 95\% CI 0.51$0.95, \mathrm{P}=0.02)$, cardiovascular death (RR $0.71,95 \% \mathrm{CI} 0.53-0.95, \mathrm{P}=0.02)$, myocardial infarction (RR $0.53,95 \%$ CI 0.42-0.67, $\mathrm{P}<0.0001$ ), stroke (RR 0.64, 95\% CI 0.41-0.98, $\mathrm{P}=0.04$ ), stent thrombosis (RR 0.63, 95\% CI 0.43$0.91, \mathrm{P}=0.01$ ) and targeted vessel revascularization ( $\mathrm{RR} 0.79$, 95\% CI $0.67-0.92, \mathrm{P}=0.003$ ). There was no significant difference in any bleeding events between the two groups. As a result of the subgroup analyses, the genotype-guided treatment was more likely to reduce the incidence of MACEs in the subgroup where the proportion of patients with ACS was $\geq 90 \%$, and subgroup of the Chinese population. Conclusion: Genotypeguided antiplatelet treatment could reduce the risk of MACEs without increasing the risk of bleeding events as compared with the standard treatment in patients with CAD or those undergoing PCI. In addition, Genotypeguided antiplatelet treatment might benefit Chinese population or patients with ACS.
\end{abstract}

\section{INTRODUCTION}

Currently, clopidogrel is a classical P2Y12 receptor inhibitor which is most commonly utilized in patients with the acute coronary syndrome (ACS) or stable coronary artery disease (CAD). Dual antiplatelet therapy (DAPT), P2Y12 inhibitor combined with aspirin, is a conventional treatment in patients undergoing percutaneous coronary intervention (PCI), which effectively reduces the risk of adverse cardiovascular events (1-3). However, the clopidogrel-induced antiplatelet effects are not adequate in almost one-fourth of patients (4). Based on the latest guidelines $(2,3,5)$, potent platelet inhibitors (ticagrelor and prasugrel) are superior in patients with myocardial infarction because of more substantial antiplatelet effects (6-8). Nonetheless, potent platelet inhibitors may increase the risk of bleeding complications compared with clopidogrel (6-9). Besides, several studies also reported more frequent discontinuation of ticagrelor due to its side effects, such as dyspnea $(6,10,11)$.

Clopidogrel is a prodrug that is transformed into an active metabolite dependent on hepatic cytochrome P450 (CYP) enzymes and inhibits platelet aggregation by inhibiting the $\mathrm{P} 2 \mathrm{Y} 12$ receptor $(12,13)$. CYP2C19 is an essential determinant affecting metabolic steps of clopidogrel of the individual response variability in clopidogrel treatment $(12,13)$. Previous studies proposed that the loss-of-function (LOF) CYP2C19*2 and CYP2C19*3 alleles were associated with the high on-treatment platelet reactivity (HTPR) and increased ischemic complications furtherly (14-16). 
It was presented that the efficacy of clopidogrel was not inferior to ticagrelor and prasugrel in patients without LOF alleles $(17,18)$.

Several published systematic reviews and meta-analyses have summarized randomized controlled trials (RCTs) and non-RCTs evidence addressing the more substantial efficacy of CYP2C19 genotype-guided antiplatelet therapy versus standard therapy (using clopidogrel or ticagrelor all the time without selection based on genotype) (19-21). In the present work we included three new studies, including the TAILOR-PCI (22) which is the largest RCT regarding CYP2C19 genotype-guided antiplatelet therapy in which the increased bleeding risk in the genotype-guided group have been reported among the primary analysis cohort. Moreover, the latest consensus in the AsiaPacific region stated that, despite the high prevalence of CYP2C19 polymorphisms in the Asia-Pacific region, genotype-guided DAPT was not recommended because of the lack of prospective randomized trials demonstrating a clinical benefit. We have noticed the emergence of new evidence from the Chinese population recently $(23,24)$. Thus, we conducted an updated meta-analysis to evaluate if the efficacy and safety of genotype-guided strategy were superior to standard therapy. In addition, we used the 2019's definitions of bleeding events as clarified by Bleeding Academic Research Consortium (BARC) and Thrombolysis in Myocardial Infarction (TIMI) standard in this systematic review and meta-analysis (25).

\section{METHODS}

\section{Protocol and search strategy}

We followed the PRISMA guideline to ensure the quality of this systematic review and meta-analysis. Two authors (B.R.T and X.W) conducted a systematical search in four electronic databases, including PubMed, EMBASE, Cochrane Central Register of Controlled Trials databases and Web of Science from inception to October 11th, 2021, without language restrictions. Reference lists of all selected articles were searched manually to identify additional studies. The search terms included 'genotype, polymorphism, pharmacogenetic, pharmacogenomic, variant, CYP2C19, guided, personalized, tailored, individualized, antiplatelet, antithrombosis, clopidogrel, ticagrelor, prasugrel, acute coronary syndromes, percutaneous coronary interventions. The full search strategy was presented in Appendix supplement.

\section{Eligibility criteria}

We used the PICOS model to select our study population. Inclusion criteria: (1) Patients: The patients with CAD or undergoing PCI planned DAPT (2) Intervention and comparison: Studies compared genotype-guided antiplatelet treatment to standard treatment; (3) Outcomes: Studies reported clinical outcomes including MACEs, all-cause mortality, cardiovascular mortality, myocardial infarction (MI), stroke, targeted vessel revascularization (TVR), stent thrombosis (ST) and bleeding events; (4) Study design: RCTs. Exclusion criteria: (1) Clinical outcomes were unavailable or insufficient; (2) Study was not published in English; (3) Data duplications.

\section{Study outcomes}

The primary efficacy outcome was MACE, which was a composite endpoint with varying definitions in different studies. We followed the definition of MACE as per each study. The secondary endpoints included all-cause mortality, cardiovascular mortality, MI, stroke, ST, and TVR.

The differences in bleeding events definition could cause heterogeneity in the pooled analysis. Bleeding events (BARC type 2,3,5), an accepted definition of any bleeding events, were regarded as the primary safety outcome. The secondary endpoints were bleeding events (BARC type 3,5), major bleeding events (TIMI) and minor bleeding events (TIMI) $(25,26)$.

\section{Selection of studies and data extraction}

Two reviewers (B.R.T and X.W) independently evaluated titles and abstracts. Duplications were removed by Endnote and manually. Any disagreement was resolved by discussion until consensus reached or by involving a third author (Z.M). Data were extracted independently from all full-text eligible articles by two reviewers. The following data were extracted: the first author, publication year, study location, study design, study period, sample size, mean age, follow-up duration, genotype test system, genotype alleles tested, treatment strategies, the proportion of LOF allele carriers, and outcomes.

\section{Quality assessment}

Two independent reviewers (B.R.T and X.W) evaluated the methodological quality of included RCTs according to the Cochrane Collaboration's tool. The risk of bias in the RCTs was evaluated based on six domains, including selection bias (random 
sequence generation and allocation concealment), performance bias (blinding of participants and personnel), detection bias (blinding of outcome assessors), attrition bias (incomplete outcome data), reporting bias (selective reporting), and other bias. Any disagreements were resolved by a third reviewer (Z.M) until a consensus reached.

\section{Statistical analysis}

The meta-analysis was performed by the Review Manager software (Revman), version 5.4 Windows. Pooled risk ratio (RR) and $95 \%$ confidence interval (CI) were calculated for each outcome. All comparisons were based on two-tailed tests, and Pvalue $<0.05$ were considered statistically significant. The heterogeneity was assessed using Cochran's Q test and I2 statistic. Significant statistical heterogeneity was defined by a $\mathrm{P}<0.1$ or $\mathrm{I} 2>50 \%$. When the P-value was $\geq 0.1$ or $\mathrm{I} 2 \leq 50 \%$, the fixedeffect model was used. If significant heterogeneity existed, the random-effect model was selected, and sensitivity analysis was conducted by removing the studies one by one in order to evaluate the potential influence of individual study on the pooled data. Subgroup analyses were performed according to indication, follow-up duration, ethnicity, proportion of LOF allele carriers, antiplatelet strategies in standard treatment groups and different genotype test system. Publication bias was examined with a funnel plot for the primary outcome.

\section{RESULTS}

\section{Study selection and characteristics}

A total of 2583 studies were identified. After removing duplications and excluding the ineligible records manually, 109 studies were eligible for full text assessment. Finally, a total of 11740 patients from eleven studies were included in the metaanalysis (Fig. 1) (22-24,27-34), of which 5958 patients $(50.75 \%)$ received genotype-guided treatment and 5782 patients $(49.25 \%)$ received standard treatment. The characteristics of the included studies were presented in Table 1a -c.

The patients in eight studies had ACS or were undergoing PCI for $\mathrm{CAD}$, and the patients with elective PCI for stable CAD were enrolled in only one study. All of the included studies tested CYP2C19*2, and some of the studies tested other variants (CYP2C19*1, CYP2C19*3, CYP2C19*17 or $\mathrm{ABCB} 1)$ by various point-of-care systems
(Spartan RX, ST Q3, Verigene, etc.). The outcomes definition and specific treatment strategy in both groups were presented in Table 1c.

\section{Study quality assessment}

The risk of biases of the included studies were evaluated and were summarized in Fig. Appendix 1. Generally, high risks of performance biases were identified, owing to the open-label design of patients and personnel. However, the included studies could meet the requirement for meta-analysis.

\section{Efficacy outcomes}

All of the included studies compared the efficacy outcomes between the genotype-guided group (GENE group) and standard treatment group (STD group). The overall results of the meta-analysis were shown in Table 2 and the forest plots of efficacy outcomes were shown in Fig. 2 and Fig. 3. Eleven studies $(n=10740)$ reported the risk of MACE. Compared with the STD group, the meta-analysis showed that the GENE group had a significantly lower risk of MACE (RR 0.60, 95\%, CI 0.44-0.82, $\mathrm{P}=0.001, \mathrm{I}^{2}=67 \%$ ). The risks of secondary outcomes were also significantly lower in the GENE group compared with the STD group, including all-cause death (RR 0.70, 95\% CI 0.51-0.95, $\left.\mathrm{P}=0.02, \mathrm{I}^{2}=46 \%\right)$, cardiovascular death (RR 0.71, 95\% CI 0.53-0.95, $\mathrm{P}=0.02, \mathrm{I}^{2}=42 \%$ ), myocardial infarction (RR 0.53, 95\% CI 0.42-0.67, $\left.\mathrm{P}<0.0001, \mathrm{I}^{2}=0 \%\right)$, stroke (RR $0.64,95 \%$ CI $0.41-0.98, \mathrm{P}=0.04, \mathrm{I}^{2}=0 \%$ ), stent thrombosis (RR $0.63,95 \%$ CI $0.43-0.91, \mathrm{P}=0.01$, I2 $=0 \%)$ and targeted vessel revascularization (RR 0.79, $95 \%$ CI $0.67-0.92, \mathrm{P}=0.003, \mathrm{I}^{2}=62 \%$ ).

\section{Safety outcomes}

Eleven studies investigated the safety outcomes between the two groups. The results of meta-analysis were shown in Table 2 and the forest plots of safety outcomes were presented in Fig. 4. The metaanalysis of eight studies $(\mathrm{n}=10605)$ showed no significant difference in terms of BARC 2,3,5 bleeding events (RR 0.87, 95\% CI 0.73-1.04, P = $0.13, \mathrm{I}^{2}=33 \%$ ). Besides, no significant differences were observed in the risks of BARC 3,5 bleeding events, major bleeding events (TIMI) and minor bleeding events (TIMI) between GENE group and STD group (RR 1.14, 95\% CI 0.82-1.58, $\mathrm{P}=0.44, \mathrm{I}^{2}$ $=0 \% ; \mathrm{RR} 1.05,95 \% \mathrm{CI} 0.68-1.63, \mathrm{P}=0.81, \mathrm{I} 2=0 \%$; RR 1.04, 95\% CI $0.64-1.67, \mathrm{P}=0.88, \mathrm{I}^{2}=0 \%$, respectively). 
Abbreviations: GENE: genotype-guided; STD: standard treatment; SD: standard deviation; PCI: percutaneous coronary intervention; ACS: acute coronary syndromes; CAD: coronary artery disease; MD: maintenance dose; LOF: loss-of-function; P: prasugrel; C: clopidogrel; T: ticagrelor; Cil: cilostazol; LD: loading dose; STEMI: ST-segment elevation myocardial infarction; NSTEMI: non-ST elevation myocardial infarction; UA: unstable angina; PLATO: Platelet Inhibition and Patient Outcomes; MACEs: major adverse cardiovascular events; HTPR: high on-treatment platelet reactivity; MI: myocardial infarction; TVR: target-vessel revascularization; ST: stent thrombosis; PRU: P2Y12 reaction unit; *1: CYP2C19*1; *2: CYP2C19*2; *3: CYP2C19*3; TIMI: Thrombolysis In Myocardial Infarction; BARC: Bleeding Academic Research Consortium.

Table 1a. Characteristics of included studies

\begin{tabular}{lllllcc}
\hline Source & Location & Ethnicity (\%) & Design & $\begin{array}{l}\text { Indication (proportion of } \\
\text { patients with ACS) }\end{array}$ & $\begin{array}{c}\text { Follow-up } \\
\text { duration }\end{array}$ & $\begin{array}{c}\text { Total number } \\
\text { Genotype }\end{array}$ \\
Standard
\end{tabular}

Table 1b. Characteristics of included studies

\begin{tabular}{|c|c|c|c|c|c|}
\hline \multirow[t]{2}{*}{ Source } & \multicolumn{2}{|c|}{ Age, mean (SD) } & \multirow[t]{2}{*}{ Genotype test system and alleles tested } & \multicolumn{2}{|c|}{$\begin{array}{c}\text { Proportion of LOF allele } \\
\text { carriers }\end{array}$} \\
\hline & $\begin{array}{l}\text { GENE } \\
\text { group }\end{array}$ & STD group & & $\begin{array}{l}\text { GENE } \\
\text { group }\end{array}$ & $\begin{array}{l}\text { STD } \\
\text { group }\end{array}$ \\
\hline Shi, $2021(23)$ & $59.7(9.6)$ & $59.8(10.4)$ & $\begin{array}{l}\text { Fluorescence in situ hybridization (TL988A, } \\
\text { Xi'an TianLong) }\end{array}$ & $57.20 \%$ & NA \\
\hline Al-Rubaish, 2021 (34) & $56.74(11.84)$ & $\begin{array}{l}55.47 \\
(11.22)\end{array}$ & $\begin{array}{l}\text { Spartan RX system } \\
\text { (Spartan Bioscience Inc.) }\end{array}$ & $31 \%$ & NA \\
\hline Zhang et al, 2020 (24) & $63.6(10.7)$ & $64.6(10.7)$ & $\begin{array}{l}\text { kit from Sinochips Bioscience Co. } \\
\text { CYP2C19*2, *3 }\end{array}$ & $51.80 \%$ & NA \\
\hline Pereira, 2020, TAILOR-PCI (22) & 62 & 62 & $\begin{array}{l}\text { Spartan Rx \& ABI TaqMan } \\
\text { CYP2C19*1, } * 2, * 3\end{array}$ & $34.20 \%$ & $35.90 \%$ \\
\hline Tuteja, 2020, ADAPT-PCI (27) & $63.0(9.7)$ & $62.9(10.2)$ & Spartan Rx. CYP2C19*1, *2, *3, *17 & $28 \%$ & NA \\
\hline Claassens, 2019, POPular (28) & $61.9(11.1)$ & $61.4(11.5)$ & Spartan Rx. CYP2C19*1, *2, *3 & $31.40 \%$ & NA \\
\hline $\begin{array}{l}\text { Notarangelo,2018, PHARMCLO } \\
\text { (29) }\end{array}$ & $71.1(12.3)$ & $70.7(12.1)$ & ST Q3. ABCB1, CYP2C19*2, *17 & $23.50 \%$ & NA \\
\hline $\begin{array}{l}\text { Tomaniak, 2017, ONSIDE TEST } \\
\text { (30) }\end{array}$ & $61.8(10.6)$ & $62.3(7.6)$ & Spartan Rx. CYP2C19*2 & $26.50 \%$ & $11.60 \%$ \\
\hline Tam, 2017 (31) & $61.6(11.8)$ & $60.3(12.2)$ & Verigene. CYP2C19*2, *3, *17 & $61.00 \%$ & $52.00 \%$ \\
\hline Xie, 2013, IAC-PCI (32) & $57.9(10.7)$ & $57.8(10.3)$ & $\begin{array}{l}\text { Shanghai Baiao technology. } \\
\text { CYP2C19*1, *2, *3 }\end{array}$ & $52.50 \%$ & NA \\
\hline $\begin{array}{l}\text { Roberts, 2012, RAPID GENE } \\
\text { (33) }\end{array}$ & $59.5(9.3)$ & $60.8(8.7)$ & Spartan Rx. CYP2C19*2 & $25 \%$ & $24 \%$ \\
\hline
\end{tabular}


Table 1c. Characteristics of included studies

\begin{tabular}{|c|c|c|c|c|}
\hline Source & GENE group strategy & STD group strategy & Primary outcomes & Secondary outcomes \\
\hline Shi, 2021 (23) & $\begin{array}{l}\text { LD: C } 300 \mathrm{mg} \text { or T } 180 \mathrm{mg} \\
\text { MD: } * 1 / * 2, * 1 / * 3, * 2 / * 2, * 2 / * 3 \text { or* } 3 / * 3 \text { : } \\
\text { Recommended treatment with T } \\
90 \mathrm{mg} / \mathrm{bd} \text {. }\end{array}$ & $\begin{array}{l}\text { LD: C } 300 \mathrm{mg} \text { or T } 180 \mathrm{mg} \\
\text { MD: C } 75 \mathrm{mg} / \mathrm{d} \text { or T } \\
\text { 90mg/bd (Prescription of } \\
\text { P2Y12 inhibitors was at the } \\
\text { discretion of cardiologists). }\end{array}$ & MACCE & $\begin{array}{l}\text { All-cause death, } \\
\text { myocardial infarction, } \\
\text { stroke, urgent coronary } \\
\text { revascularization, stent } \\
\text { thrombosis, bleeding } \\
\text { events (BARC) }\end{array}$ \\
\hline $\begin{array}{l}\text { Al-Rubaish, } 2021 \\
\text { (34) }\end{array}$ & $\begin{array}{l}\text { MD: } \\
\text { Carriers of } * 2: \mathrm{T} 10 \mathrm{mg} / \mathrm{d} \\
\text { Noncarrier of } * 2: \mathrm{C} \\
75 \mathrm{mg} / \mathrm{d}\end{array}$ & MD: C $75 \mathrm{mg} / \mathrm{d}$ & $\begin{array}{l}\text { MACE, recurrent } \\
\text { myocardial infarction, } \\
\text { non-fatal stroke, } \\
\text { cardiovascular death } \\
\text { and major bleeding } \\
\text { (PLATO) }\end{array}$ & $\begin{array}{l}\text { Stroke, stent thrombosis, } \\
\text { target vessel } \\
\text { revascularization, all-cause } \\
\text { death }\end{array}$ \\
\hline $\begin{array}{l}\text { Zhang et al, } 2020 \\
\text { (24) }\end{array}$ & $\begin{array}{l}\text { Extensive metabolizers: } \mathrm{C} 75 \mathrm{mg} / \mathrm{d} \text {; } \\
\text { intermediate metabolizers: } \mathrm{C} 150 \mathrm{mg} / \mathrm{d} \text {; } \\
\text { poor metabolizers: T } 180 \mathrm{mg} / \mathrm{d} \text {. For } \\
\text { extensive metabolizers and intermediate } \\
\text { metabolizers patients with HPR, the } \\
\text { antiplatelet treatment was changed or } \\
\text { unchanged by the clinicians according to } \\
\text { the patient's conditions. }\end{array}$ & $\begin{array}{l}\text { LD: not mentioned } \\
\text { MD: C } 75 \mathrm{mg} / \mathrm{d}\end{array}$ & $\begin{array}{l}\text { Safety outcome: } \\
\text { moderate or severe } \\
\text { hemorrhage; } \\
\text { Efficacy outcome: } \\
\text { MACEs, MI, ST, all- } \\
\text { cause death }\end{array}$ & $\begin{array}{l}\text { Safety outcome: mild } \\
\text { hemorrhage }\end{array}$ \\
\hline $\begin{array}{l}\text { Pereira, 2020, } \\
\text { TAILOR-PCI } \\
(22)\end{array}$ & $\begin{array}{l}\text { LD: the choice was at the discretion of } \\
\text { the treating physician. MD: CYP2C19 } \\
\text { LOF carriers: T; noncarriers or those with } \\
\text { inconclusive results: C. Prasugrel was } \\
\text { recommended as an alternative for } \\
\text { patients who did not tolerate ticagrelor. }\end{array}$ & $\begin{array}{l}\text { LD: the choice at the } \\
\text { discretion of the treating } \\
\text { physician. } \\
\text { MD: C: according to drug } \\
\text { label instructions. }\end{array}$ & $\begin{array}{l}\text { Cardiovascular death, } \\
\text { MI, stroke, ST }\end{array}$ & $\begin{array}{l}\text { TIMI major or minor } \\
\text { bleeding, TIMI major } \\
\text { bleeding, BARC bleeding, } \\
\text { all-cause death, }\end{array}$ \\
\hline $\begin{array}{l}\text { Tuteja, 2020, } \\
\text { ADAPT-PCI (27) }\end{array}$ & $\begin{array}{l}\text { Slow metabolizer status [ } 1 \text { or } 2 \text { LOF } \\
\text { mutations }(* 2 \text { or } * 3 \text { ) in CYP2C19]: P or } \\
\text { T. Normal metabolizer status } \\
\text { (homozygous for the } * 1 \text { allele in } \\
\text { CYP2C19): C. Antiplatelet choice is } \\
\text { ultimately decided by physician judgment } \\
\text { incorporating all clinical factors. }\end{array}$ & $\begin{array}{l}\text { Choice of antiplatelet } \\
\text { therapy will be decided by } \\
\text { treating physician as per } \\
\text { usual care }\end{array}$ & $\begin{array}{l}\text { Proportion of } \\
\text { participants receiving } \\
\text { prasugrel/ticagrelor }\end{array}$ & $\begin{array}{l}\text { MACEs: cardiovascular } \\
\text { mortality, MI, stroke, ST, } \\
\text { and urgent } \\
\text { revascularization; } \\
\text { Safety outcomes: BARC } \\
\text { bleeding }\end{array}$ \\
\hline $\begin{array}{l}\text { Claassens, 2019, } \\
\text { POPular (28) }\end{array}$ & $\begin{array}{l}\text { MD: Carry } 1 \text { or more CYP } 2 \text { C } 19 * 2 \text { or } * 3 \\
\text { LOF alleles: T } 90 \mathrm{mg} / \mathrm{bd} \text { or P } \\
10 \mathrm{mg} / \mathrm{d} \text { (patients }>75 \text { years or weighing } \\
<60 \mathrm{~kg} \text { will receive } 5 \mathrm{mg} \text { ); Noncarriers: } \\
\text { C } 75 \mathrm{mg} / \mathrm{d} \text {. }\end{array}$ & $\begin{array}{l}\text { MD: the first } 100 \text { patients: } \\
\text { C } 75 \mathrm{mg} / \mathrm{d} \text {. After February } \\
2012, \mathrm{~T} 90 \mathrm{mg} / \mathrm{bd} \text { or } \mathrm{P} \\
10 \mathrm{mg} / \mathrm{d}(\text { patients }>75 \text { years } \\
\text { or weighing }<60 \mathrm{~kg} \text { will } \\
\text { receive } 5 \mathrm{mg} \text { ). }\end{array}$ & $\begin{array}{l}\text { Net adverse clinical } \\
\text { events: death, MI, ST, } \\
\text { stroke. } \\
\text { Safety outcomes: } \\
\text { major bleeding } \\
\text { (PLATO criteria) }\end{array}$ & $\begin{array}{l}\text { Safety outcomes: PLATO } \\
\text { major bleeding or minor } \\
\text { bleeding, BARC } 3 \text { to } 5 \\
\text { defined major bleeding }\end{array}$ \\
\hline $\begin{array}{l}\text { Notarangelo,2018, } \\
\text { PHARMCLO } \\
\text { (29) }\end{array}$ & $\begin{array}{l}\text { Based on the combination of genotypes } \\
\text { ABCB } 13435, \text { CYP2C } 19 * 2 \text { and } * 17\end{array}$ & $\begin{array}{l}\text { Based on clinical } \\
\text { characteristics and the } \\
\text { clinicians' preference }\end{array}$ & $\begin{array}{l}\text { MACEs: death, MI, } \\
\text { stroke, ST }\end{array}$ & $\begin{array}{l}\text { Safety outcomes: BARC } 3 \\
\text { to } 5 \text { defined major bleeding }\end{array}$ \\
\hline $\begin{array}{l}\text { Tomaniak, 2017, } \\
\text { ONSIDE TEST } \\
\text { (30) }\end{array}$ & $\begin{array}{l}\mathrm{MD}: * 1 / * 1: \mathrm{C} 75 \mathrm{mg} / \mathrm{d} ; * 1 / * 2: \mathrm{P}(60 \mathrm{mg}) \\
2 \mathrm{~h} \text { before PCI then } 10 \mathrm{mg} / \mathrm{d}\end{array}$ & MD:C $75 \mathrm{mg} / \mathrm{d}$ & $\begin{array}{l}\text { MACEs: death, MI, } \\
\text { stroke, ST }\end{array}$ & $\begin{array}{l}\text { Safety outcomes: BARC } 3 \\
\text { to } 5 \text { defined major bleeding }\end{array}$ \\
\hline Tam, 2017 (31) & $\begin{array}{l}\text { LD: } * 1 / * 1: \text { C } 300 \mathrm{mg} . * 1 / * 2 \text { or } * 1 / * 3: \mathrm{C} \\
600 \mathrm{mg} \\
* 2 / * 2, * 2 / * 3 \text { or } * 3 / * 3: \text { C } 600 \mathrm{mg} / \mathrm{d}+\mathrm{Cil} \\
200 \mathrm{mg} \\
\text { MD: } * 1 / * 1: \mathrm{C} 75 \mathrm{mg} / \mathrm{d} . * 1 / * 2 \text { or } * 1 / * 3: \mathrm{C} \\
150 \mathrm{mg} / \mathrm{d} \\
* 2 / * 2, * 2 / * 3 \text { or } * 3 / * 3: \mathrm{C} 150 \mathrm{mg} / \mathrm{d}+\mathrm{Cil} \\
100 \mathrm{mg} / \mathrm{bd}\end{array}$ & $\begin{array}{l}\text { LD: C } 600 \mathrm{mg} \text { (PCI for } \\
\text { STEMI), } 300 \mathrm{mg} \text { (PCI for } \\
\text { NSTEMI or UA, or STEMI } \\
\text { without PCI) } \\
\text { MD: C } 75 \mathrm{mg} / \mathrm{d}\end{array}$ & HTPR: PRU > 208 & $\begin{array}{l}\text { MACEs: mortality, MI, and } \\
\text { stroke }\end{array}$ \\
\hline $\begin{array}{l}\text { Xie, 2013, IAC- } \\
\text { PCI (32) }\end{array}$ & $\begin{array}{l}\text { LD: } * 1 / * 1: \text { C } 300 \mathrm{mg} . * 1 / * 2 \text { or } * 1 / * 3: \mathrm{C} \\
600 \mathrm{mg} \\
* 2 / * 2, * 2 / * 3 \text { or } * 3 / * 3: \mathrm{C} 600 \mathrm{mg} / \mathrm{d}+\mathrm{Cil} \\
200 \mathrm{mg} \\
\mathrm{MD}: * 1 / * 1: \mathrm{C} 75 \mathrm{mg} / \mathrm{d} . * 1 / * 2 \text { or } * 1 / * 3: \mathrm{C} \\
150 \mathrm{mg} / \mathrm{d} \\
* 2 / * 2, * 2 / * 3 \text { or } * 3 / * 3: \mathrm{C} 150 \mathrm{mg} / \mathrm{d}+\mathrm{Cil} \\
100 \mathrm{mg} / \mathrm{bd}\end{array}$ & $\begin{array}{l}\text { LD: C } 300 \mathrm{mg} \\
\text { MD:C } 75 \mathrm{mg} / \mathrm{d}\end{array}$ & $\begin{array}{l}\text { MACEs: mortality, } \\
\text { MI, stroke, TVR }\end{array}$ & $\begin{array}{l}\text { MACEs } \\
\text { Safety outcomes: BARC } \\
\text { defined all bleeding }\end{array}$ \\
\hline $\begin{array}{l}\text { Roberts, 2012, } \\
\text { RAPID GENE } \\
\text { (33) }\end{array}$ & $\begin{array}{l}\text { MD: Carriers of *2: P } 10 \mathrm{mg} / \mathrm{d} \text {. } \\
\text { Noncarrier of } * 2: \text { C: } 75 \mathrm{mg} / \mathrm{d}\end{array}$ & MD: C $75 \mathrm{mg} / \mathrm{d}$ & HTPR: PRU > 208 & $\begin{array}{l}\text { MACEs: Mortality, MI, ST } \\
\text { Safety outcomes: TIMI } \\
\text { major and minor bleeding }\end{array}$ \\
\hline
\end{tabular}


Table 2. Results of the meta-analysis for efficiency outcomes and safety outcomes between GENE group and STD group

\begin{tabular}{|c|c|c|c|c|c|c|c|c|}
\hline \multirow[b]{2}{*}{ Event } & \multirow{2}{*}{$\begin{array}{l}\text { Number of } \\
\text { studies }\end{array}$} & \multirow[b]{2}{*}{ Participants } & \multicolumn{2}{|l|}{ Events } & \multirow{2}{*}{$\begin{array}{l}\text { Pooled RR } \\
\text { (95\% CI) }\end{array}$} & \multirow[b]{2}{*}{ P-value } & \multicolumn{2}{|c|}{ Heterogeneity } \\
\hline & & & GENE & STD & & & I2 (\%)* & P-value \\
\hline \multicolumn{9}{|l|}{ Efficiency outcomes } \\
\hline MACEs & 11 & 11740 & 286 & 407 & $0.60(0.44,0.82)$ & 0.001 & $67 \%$ & 0.001 \\
\hline All-cause mortality & 10 & 10852 & 66 & 91 & $0.70(0.51,0.95)$ & 0.02 & $46 \%$ & 0.07 \\
\hline Cardiovascular mortality & 9 & 11307 & 73 & 99 & $0.71(0.53,0.95)$ & 0.02 & $42 \%$ & 0.10 \\
\hline MI & 11 & 11740 & 102 & 186 & $0.53(0.42,0.67)$ & $<0.00001$ & $0 \%$ & 0.53 \\
\hline Stroke & 9 & 11063 & 33 & 49 & $0.64(0.41,0.98)$ & 0.04 & $0 \%$ & 0.94 \\
\hline ST & 10 & 11608 & 46 & 65 & $0.63(0.43,0.91)$ & 0.01 & $0 \%$ & 0.62 \\
\hline TVR & 6 & 4640 & 197 & 215 & $0.79(0.67,0.92)$ & 0.003 & $62 \%$ & 0.03 \\
\hline \multicolumn{9}{|l|}{ Safety outcomes } \\
\hline $\begin{array}{l}\text { Bleeding events (BARC } \\
\text { type } 2,3,5 \text { ) }\end{array}$ & 8 & 10605 & 220 & 248 & $0.87(0.73,1.04)$ & 0.13 & $33 \%$ & 0.17 \\
\hline $\begin{array}{l}\text { Bleeding events (BARC } \\
\text { type } 3,5 \text { ) }\end{array}$ & 5 & 8458 & 74 & 65 & $1.14(0.82,1.58)$ & 0.44 & $0 \%$ & 0.90 \\
\hline $\begin{array}{l}\text { Major bleeding events } \\
\text { (TIMI) }\end{array}$ & 3 & 7951 & 41 & 39 & $1.05(0.68,1.63)$ & 0.81 & $0 \%$ & 0.80 \\
\hline $\begin{array}{l}\text { Minor bleeding events } \\
\text { (TIMI) }\end{array}$ & 3 & 7951 & 34 & 33 & $1.04(0.64,1.67)$ & 0.88 & $0 \%$ & 0.88 \\
\hline
\end{tabular}

CI, confidence interval; GENE, genotyping-guided treatment group; STD, standard treatment group; MACEs, major adverse cardiovascular events; MI, myocardial infarction; RR, risk ratio; ST, stent thrombosis; TVR, targeted vessel revascularization; BARC, Bleeding Academic Research Consortium; TIMI, Thrombolysis In Myocardial Infarction. *I2 $\geqslant$ $50 \%$ in pooled analysis of MACEs, the random effects model was used.

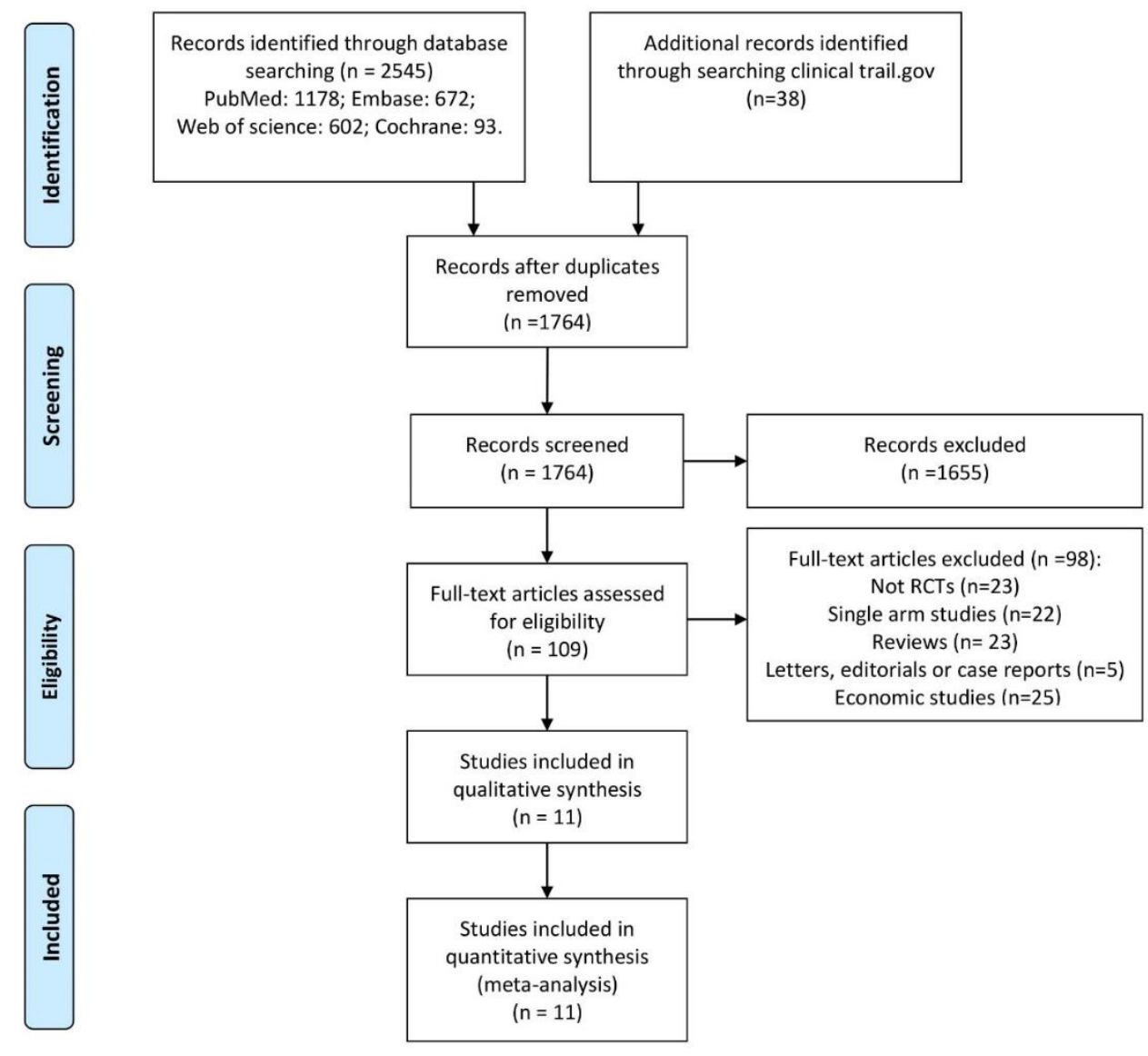

Figure 1. The flow diagram of study selection 


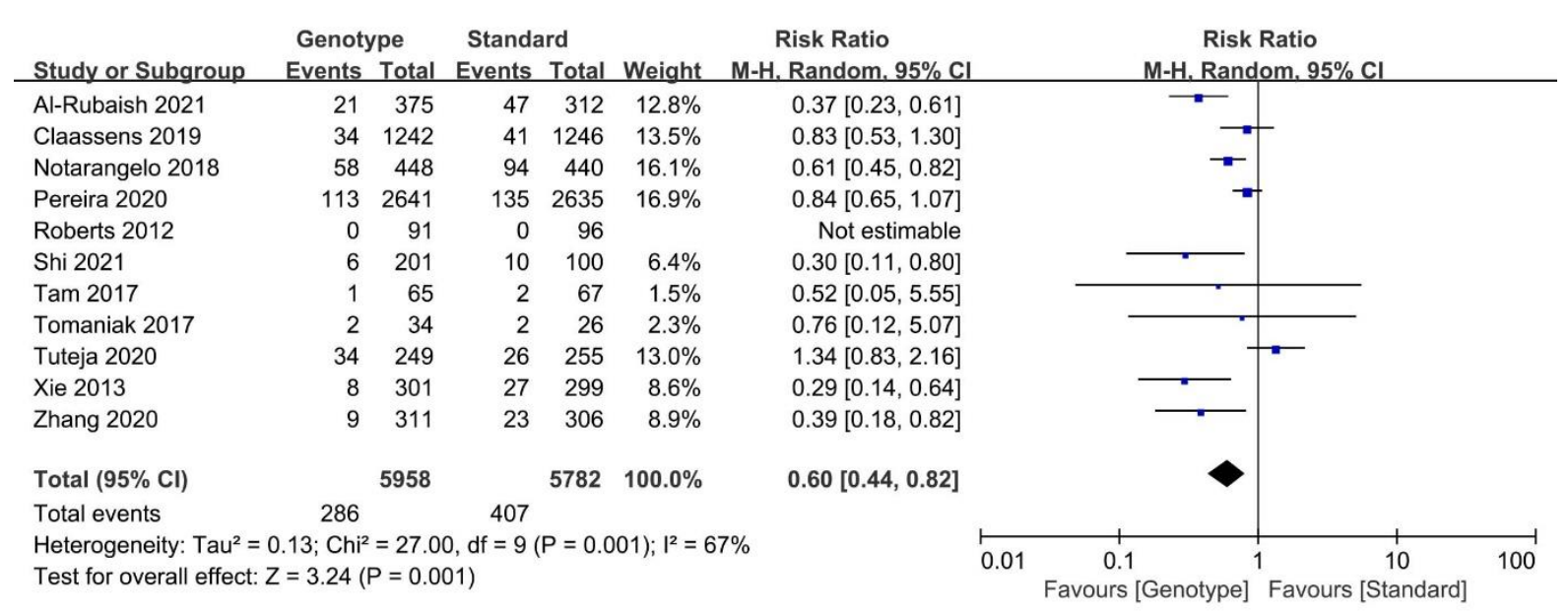

Figure 2. The forest plot of MACE

\section{Sensitivity analysis and subgroup analysis}

The sensitivity analysis was conducted in the primary efficacy outcome, which showed a significant heterogeneity in the meta-analysis $\left(\mathrm{I}^{2}=67 \%, \mathrm{P}=\right.$ $0.001)$. The results showed that, after the removal of each single study, the heterogeneity of the remaining studies and the signification of RR did not change. Publication bias in MACEs was not detected by the funnel plot (Fig. Appendix 2) with visible symmetry in meta-analyses.

The subgroup analyses were performed for primary efficacy outcome and primary safety outcome according to the different characteristics among these included studies. The overall results of subgroup analyses for MACEs and any bleeding events were shown in Table 3 and Table 4 respectively and the forest plots of subgroup analyses were presented in Appendix supplement.

In the subgroup analyses for MACEs, when studies were classified by the proportion of patients with ACS, ethnicity, and the proportion of LOF allele carriers in GENE group, the between-subgroup heterogeneities were significant. The p-values of pooled RR were significantly lower in the subgroups with clopidogrel in STD group, ACS $\geq 90 \%$, Chinese population, and LOF allele carriers $\geq 50 \%$ in GENE group, and sample size < 200. There were no significant differences between GENE group and STD group in the other subgroups. For any bleeding events (BARC type 2,3,5), when studies were classified by the proportion of patients with ACS, the between-subgroup heterogeneities were significant. Meanwhile, GENE group had a reduced risk of any bleeding events in the subgroups with ticagrelor, prasugrel or uncertain treatment in STD group, and
$\mathrm{ACS} \geq 90 \%$.

\section{DISCUSSION}

DAPT has long been the standard of therapy in preventing cardiovascular and cerebrovascular ischemic events in patients with stable CAD and ACS undergoing PCI (1-3), but the choice of antiplatelet treatment composition was a considerable challenge for clinicians. Impaired conversion of clopidogrel to the active metabolite might be caused by LOF mutations, leading to HTPR commonly among patients on clopidogrel treatment while rarely in prasugrel users (14-16). HTPR has been consistently associated with an increased risk of ST and MACE (15). Thus, genotyping might be utility guidance for individualized P2Y12 inhibitor therapy, such as escalation (switch from clopidogrel to ticagrelor or prasugrel) or de-escalation treatment, to reduce the risk of ischemic and hemorrhagic (35). In recent years, point-of-care genotyping assays have become available in more medical institutions, enabling implementation in routine treatment.

However, the expert consensus statement from JACC and Asia-Pacific region showed that CYP2C19 genotype-guided antiplatelet strategy was not recommended, because of lack of data from dedicated studies $(36,37)$. In fact, the recent emergence of new evidence might change the results of previous meta-analyses. Therefore, the aim of the meta-analysis was to evaluate if the genotype-guided strategy was superior to standard therapy in patients with CAD or undergoing PCI. After performing the meta-analysis including eleven RCTs, we found that 
Table 3. Subgroup analysis for MACEs between GENE group and STD group

\begin{tabular}{|c|c|c|c|c|c|c|}
\hline \multirow[b]{2}{*}{ Subgroup category } & \multirow[b]{2}{*}{$\begin{array}{l}\text { Number } \\
\text { of studies }\end{array}$} & \multirow[b]{2}{*}{ Patients } & \multirow[b]{2}{*}{ Pooled RR (95\% CI) } & \multirow[b]{2}{*}{ P-value } & \multicolumn{2}{|c|}{ Heterogeneity [I2(\%)] } \\
\hline & & & & & $\begin{array}{l}\text { In- } \\
\text { subgroup }\end{array}$ & $\begin{array}{l}\text { Between- } \\
\text { subgroup }\end{array}$ \\
\hline \multicolumn{7}{|l|}{ Follow-up duration } \\
\hline$\geq 12$ months & 8 & 10821 & $0.65(0.47,0.89)$ & $0.007 *$ & $68 \%$ & \multirow{2}{*}{$69.4 \%$} \\
\hline$<12$ months & 3 & 919 & $0.31(0.15,0.65)$ & $0.002 *$ & $0 \%$ & \\
\hline \multicolumn{7}{|l|}{ Treatment strategy in STD group } \\
\hline Clopidogrel & 7 & 7559 & $0.48(0.29,0.79)$ & $0.004 *$ & $67 \%$ & \multirow{3}{*}{$22.1 \%$} \\
\hline Ticagrelor, Prasugrel & 1 & 2488 & $0.83(0.53,1.30)$ & 0.42 & - & \\
\hline Uncertain & 3 & 1693 & $0.68(0.34,1.38)$ & 0.75 & $87 \%$ & \\
\hline \multicolumn{7}{|l|}{ Proportion of patients with ACS } \\
\hline$\geq 90 \%$ & 7 & 5713 & $0.52(0.43,0.64)$ & $<0.00001 *$ & $45 \%$ & \multirow{2}{*}{$92.8 \%$} \\
\hline$<90 \%$ & 4 & 6027 & $0.91(0.74,1.13)$ & 0.41 & $33 \%$ & \\
\hline \multicolumn{7}{|l|}{ Ethnicity } \\
\hline Caucasian & 5 & 9343 & $0.83(0.63,1.11)$ & 0.21 & $62 \%$ & \multirow{2}{*}{$90.8 \%$} \\
\hline Chinese & 4 & 1650 & $0.33(0.21,0.53)$ & $<0.00001 *$ & $0 \%$ & \\
\hline \multicolumn{7}{|c|}{$\begin{array}{l}\text { Proportion of LOF allele carriers in } \\
\text { GENE group }\end{array}$} \\
\hline$<50 \%$ & 7 & 10090 & $0.73(0.53,1.01)$ & 0.06 & $69 \%$ & \multirow{2}{*}{$86.7 \%$} \\
\hline$\geq 50 \%$ & 4 & 1650 & $0.33(0.21,0.53)$ & $<0.00001 *$ & $0 \%$ & \\
\hline \multicolumn{7}{|l|}{ Sample size } \\
\hline$\geq 200$ & 8 & 11361 & $0.60(0.43,0.83)$ & $0.002 *$ & $74 \%$ & \multirow{2}{*}{$0.0 \%$} \\
\hline$<200$ & 3 & 379 & $0.66(0.15,2.88)$ & 0.58 & $0 \%$ & \\
\hline \multicolumn{7}{|l|}{ Genotype test system } \\
\hline Spartan Rx & 6 & 9202 & $0.77(0.52,1.16)$ & 0.22 & $71 \%$ & \multirow{2}{*}{$72.5 \%$} \\
\hline the others (ST Q3, Verigene, etc) & 5 & 2538 & $0.46(0.33,0.65)$ & $<0.00001 *$ & $19 \%$ & \\
\hline
\end{tabular}

*P<0.05; ACS, acute coronary syndrome; CI, confidence interval; GENE, genotyping-guided treatment; LOF loss-offunction; RR, risk ratio; STD, standard treatment.

Table 4. Subgroup analysis for Bleeding events (BARC type 2,3,5) between GENE group and STD group

\begin{tabular}{|c|c|c|c|c|c|c|}
\hline \multirow[b]{2}{*}{ Subgroup category } & \multirow[b]{2}{*}{$\begin{array}{l}\text { Number of } \\
\text { studies }\end{array}$} & \multirow[b]{2}{*}{ Patients } & \multirow[b]{2}{*}{ Pooled RR (95\% CI) } & \multirow[b]{2}{*}{ P-value } & \multicolumn{2}{|c|}{ Heterogeneity [ I $2(\%)]$} \\
\hline & & & & & In-subgroup & $\begin{array}{l}\text { Between- } \\
\text { subgroup }\end{array}$ \\
\hline \multicolumn{7}{|l|}{ Follow-up duration } \\
\hline$\geq 12$ months & 6 & 9873 & $0.89(0.75,1.07)$ & 0.21 & $34 \%$ & \multirow{2}{*}{$24.5 \%$} \\
\hline$<12$ months & 2 & 732 & $0.50(0.19,1.32)$ & 0.16 & $41 \%$ & \\
\hline \multicolumn{7}{|c|}{ Treatment strategy in STD group } \\
\hline Clopidogrel & 5 & 7312 & $1.03(0.75,1.41)$ & 0.85 & $44 \%$ & \multirow{3}{*}{$23.4 \%$} \\
\hline Ticagrelor, Prasugrel & 1 & 2488 & $0.78(0.62,0.97)$ & $0.03 *$ & $0 \%$ & \\
\hline Uncertain & 2 & 805 & $1.10(0.57,2.10)$ & 0.78 & $0 \%$ & \\
\hline \multicolumn{7}{|c|}{ Proportion of patients with ACS } \\
\hline$\geq 90 \%$ & 6 & 4825 & $0.77(0.63,0.95)$ & $0.01 *$ & $18 \%$ & \multirow{2}{*}{$77.9 \%$} \\
\hline$<90 \%$ & 2 & 5780 & $1.18(0.85,1.64)$ & 0.33 & $0 \%$ & \\
\hline \multicolumn{7}{|c|}{$\begin{array}{l}\text { Proportion of LOF allele carriers in } \\
\text { GENE group }\end{array}$} \\
\hline$<50 \%$ & 4 & 8955 & $0.92(0.63,1.35)$ & 0.68 & $55 \%$ & \multirow{2}{*}{$0.0 \%$} \\
\hline$\geq 50 \%$ & 4 & 1650 & $0.92(0.45,1.88)$ & 0.81 & $21 \%$ & \\
\hline
\end{tabular}

$* \mathrm{P}<0.05$; ACS, acute coronary syndrome; CI, confidence interval; GENE, genotyping-guided treatment; LOF loss-offunction; RR, risk ratio; ST, standard treatment. 


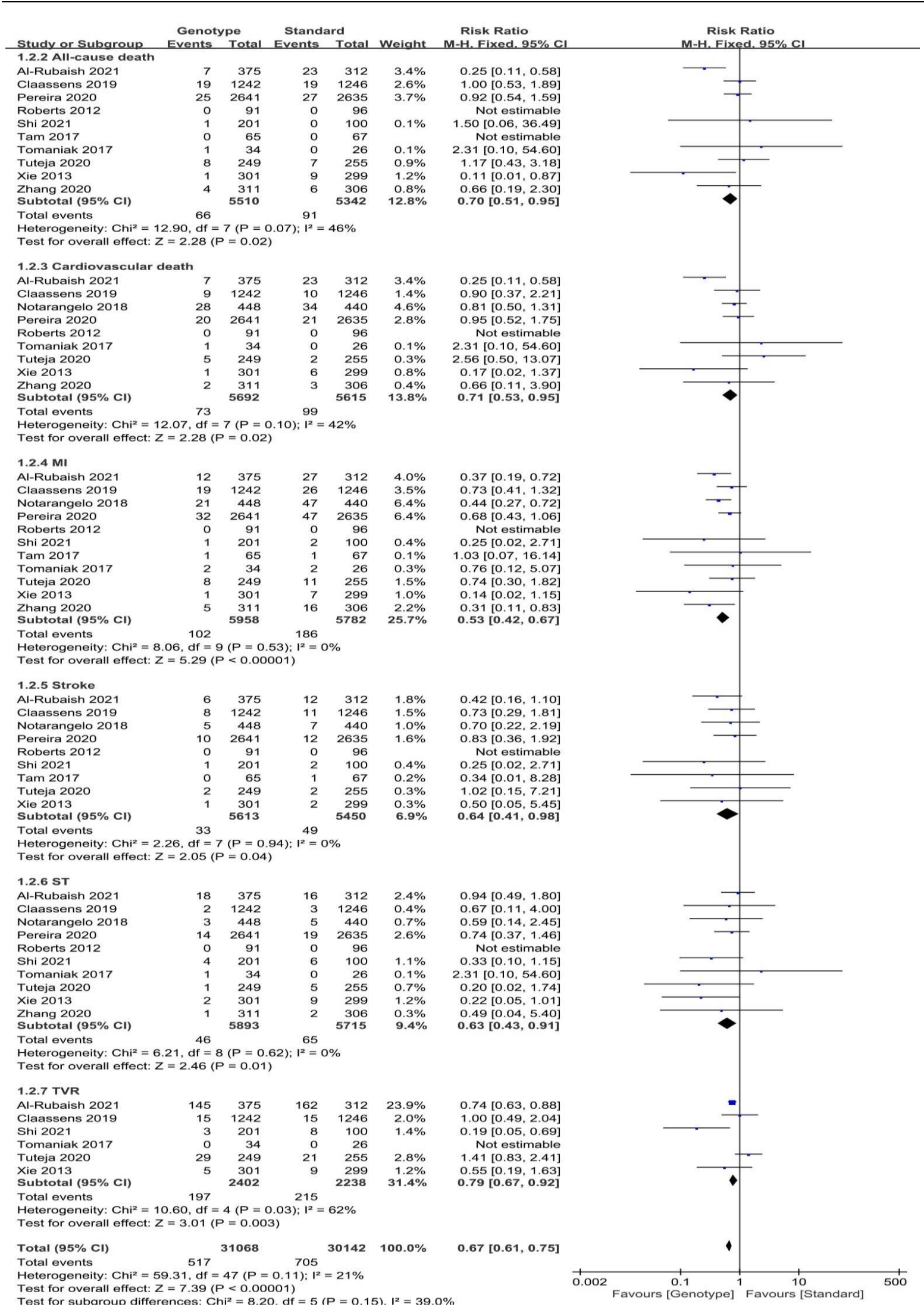

Figure 3. The forest plot of all-cause death, cardiovascular death, MI, stroke, ST, and TVR. Abbreviation: MI, myocardial infarction; TVR, targeted vessel revascularization, ST, stent thrombosis. 


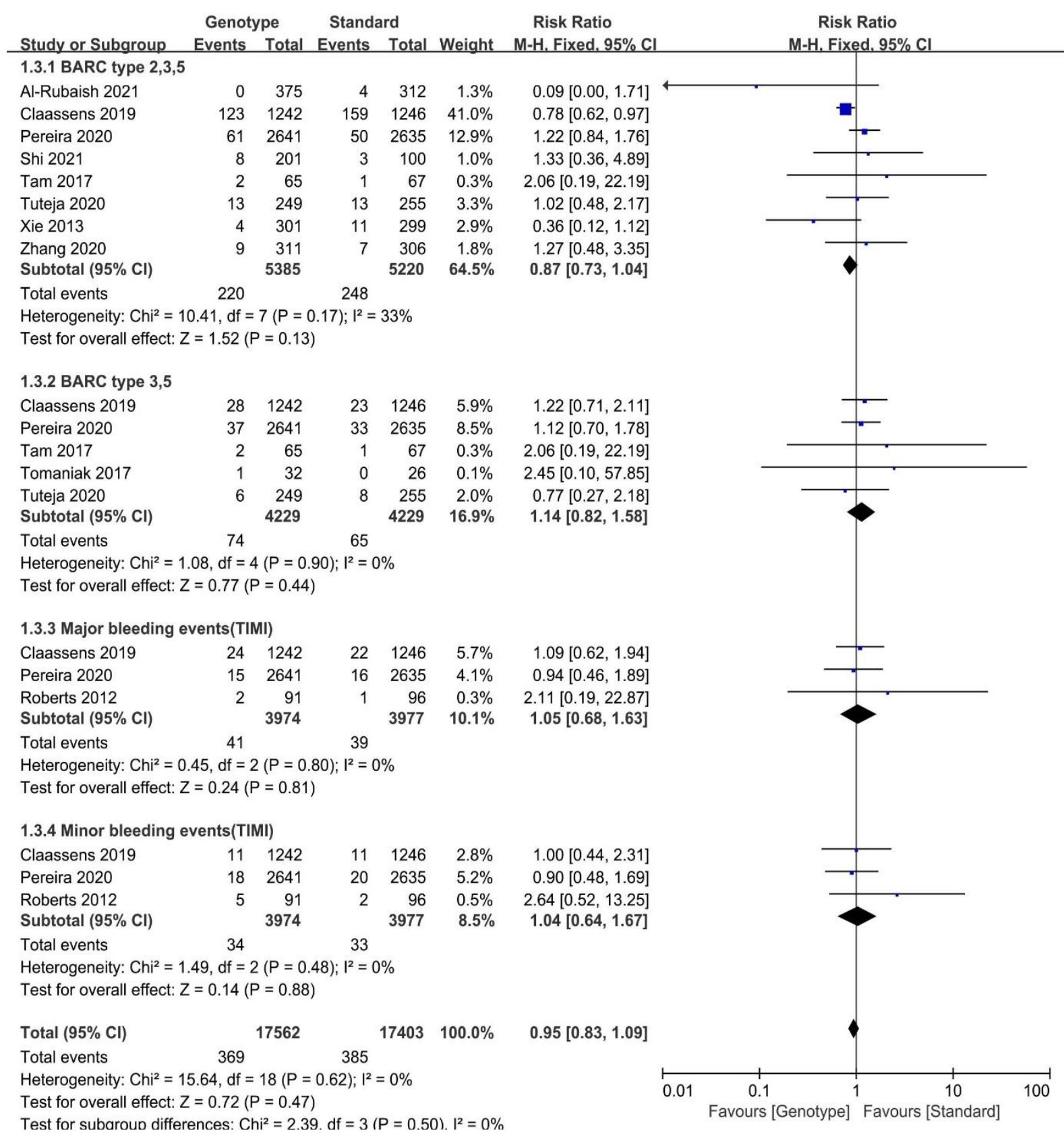

Figure 4. The forest plot of safety outcomes. Abbreviation: BARC, Bleeding Academic Research Consortium; TIMI, Thrombolysis In Myocardial Infarction.

the risk of MACEs in the GENE group was significantly lower compared with the STD group. And a significant reduction in the risk of all-cause death, cardiovascular death, MI, stroke, ST and TVR were also observed in the GENE group. Moreover, incidences of the safety endpoints were comparable between the two groups. However, the reduced risk of MACE might only occur in the Chinese patients and patients with ACS. In addition, the genotypeguided antiplatelet strategy might reduce the risk of
MACE only compared with the strategy for the fixed use of clopidogrel in STD group.

\section{Relation to prior studies and innovation}

Previous meta-analyses have drawn inconsistent conclusions about whether genotype-guided antiplatelet therapy could reduce the risk of MACE which might be caused by the different inclusion of criteria $(19,21,38)$. Our study included more trials (11 RCTs) and a substantially larger sample size 
(11740 patients), particularly including the two more trials from Asian population $(23,24)$ and the data of the whole population in the TAILOR-PCI trial (previous meta-analysis only included part of population) (20), which is the largest relevant trial and at a low risk of bias (22). The results of our study differed from previous studies in efficacy outcomes, including all-cause death, cardiovascular death, stroke, and TVR. Moreover, in previous metaanalyses, the safety outcomes, including major bleeding events and minor bleeding events, were stratified by the standard of each original study. Our study included four safety outcomes (bleeding events) classified by BARC and TIMI standard, which could avoid the bias caused by different definitions of outcomes. Additionally, subgroup analyses have been reported only for MACEs in prior metaanalyses $(20,21)$. We performed more comprehensive and more explicable subgroup analyses for both MACEs and bleeding events to explore the impact of difference among the included studies and the reason for heterogeneity.

\section{Heterogeneity}

The pooled analysis for MACEs showed a significant heterogeneity, whereas the result of sensitivity analysis with the leave-one-out method showed no effect on the heterogeneity in the outcome of MACE, which indicated that the heterogeneity did not originate from a single study. However, the different characteristics among included studies might cause the high heterogeneity in this meta-analysis, including different follow-up duration, diagnoses of enrolled patients, ethnicity of enrolled patients and proportions of LOF allele carriers among included studies, which was also confirmed by the result of subgroup analyses.

Interpretation for the result of subgroup analyses Treatment strategy in the STD group. We classified studies into "Clopidogrel", "Ticagrelor or prasugrel", and uncertain subgroup according to the choice of antiplatelet drug in the STD group. In the "Clopidogrel" subgroup, genotype-guided therapy reduced the risk of MACE. But the effect of genotype-guided therapy was not better than strategy of using fixed ticagrelor or prasugrel. Several studies proposed that the efficacy of potent P2Y12 inhibitors was prior to clopidogrel $(6,8)$. Currently, ticagrelor or prasugrel was recommended for patients after PCI without bleeding risk instead of clopidogrel in European Society of Cardiology (ESC) latest guideline (2), which has gradually become the first choice for clinicians without support by precision medicine. However, there was only one RCT where patients used ticagrelor or prasugrel as a routine treatment strategy in the control group. Patients who were tested as noncarriers of the CYP2C19 LOF allele use clopidogrel rather than potent P2Y12 inhibitors in the genotype-guided group, which can be considered as de-escalation strategy $(36,39)$. In the subgroup analysis of safety endpoints, patients who received genotype-guided therapy had a significant reduction in the risk of any bleeding events compared with the fixed ticagrelor or prasugrel group, which might be explained that genotype-guided de-escalation improved the safety of treatment. However, this result was limited by a lack of relevant studies and poor sample size. Therefore, more studies were needed to explore whether de-escalation therapy based on genotype guidance can improve the prognosis of patients.

Ethnicity. Nine studies included were divided into Caucasian and Chinese according to the ethnicity of most people. From the results of subgroup analysis, a significant reduction in the incidence of MACE was observed in Chinese subgroup between the two groups, while the Caucasian population might not benefit from genotype-guided strategy. A number of studies have shown that more Asians carried LOF allele (CYP2C19*2 and CYP2C19*3) compared with African and European populations (40-42). Nevertheless, CYP2C19*17, an increased function allele, was more likely carried in Caucasians and black populations (40-42). Diverse interracial proportion of LOF carriers could be one possible reason for the statistically difference between two subgroups.

Proportion of LOF allele carriers in GENE group. We divided studies into the " $\geq 50 \%$ " subgroup and the " $<50 \%$ " subgroup according to the proportion of LOF allele carriers in the GENE group. The results of subgroup analysis showed that genotype-guided therapy could significantly reduce the incidence of MACE in the " $\geq 50 \%$ " subgroup, but there was no significant difference found in the " $<50 \%$ " subgroup. For those who carrying the LOF gene, genotypeguided therapy could avoid the poor efficacy of clopidogrel, which might also interpret why Chinese population benefits more from the genotype-guided strategy. Therefore, for population with low rate of carrying LOF, fixed use of clopidogrel or choosing drugs based on clinical characteristics might be a more economical and reasonable choice, but 
genotype-guided strategy still had advantages for people with high rate of carrying LOF.

Proportion of ACS patients. The enrolled patients were diagnosed with different severity of CAD among the included studies, which might cause bias in meta-analysis. Patients in some of the studies were all having $\operatorname{ACS}(23,24,28,31,32,34)$, while the patients in the study ONSIDE TEST (Tomaniak,2017) were all having stable coronary heart disease (30). All studies were divided into the " $>90 \%$ " subgroup and the " $\leq 90 \%$ " subgroup according to the proportion of patients diagnosed with ACS. In the "> 90\%" subgroup, genotypeguided therapy could reduce the risk of MACE significantly, while the difference was not significant in the " $\leq 90 \%$ " subgroup between the two groups. The result might indicate that patients with ACS were more likely to benefit from genotype-guided therapy, which was reasonable for the distribution of medical resources in the real world, that is, more medical resources should be allocated to more severe patients. In latest guidelines (2), clopidogrel, instead of prasugrel or ticagrelor, was recommended for people with stable CAD after PCI. Therefore, if economic factor was considered, fixed clopidogrel treatment might be suitable as a routine DAPT strategy for patients with stable CAD. However, this result needed to be interpreted with caution due to the limitation of sample size.

Others. Various system of genotype detection may affect the results of genotyping assays. Six of the included studies used the SpartanRx system for rapid genotype detection, while five studies used other systems (ST Q3, Verigene, etc.). In TAILOR-PCI (22), the results of rapid genotyping assays by SpartanRx were verified by the TaqMan system to ensure accuracy, while results in other studies were not verified by the gold standard method (real-time polymerase chain reaction). We divided the included study into two subgroups according to the method of the gene detection system. The reduced heterogeneity indicated that the different methods of genotyping assays might be one of the sources of heterogeneity.

In terms of sample size, three of the included studies were conducted in a single center with a small sample size, and the conclusions from those might not be convincing enough. The results of subgroup analysis based on the sample size showed that genotype-guided therapy could not reduce the incidence of MACEs significantly in studies with less than 200 patients. However, a significant reduction of risk of MACEs was found in the subgroup of large sample size, and the inclusion of three small-scale studies did not change the significance of pooled RR.

Besides, as shown in the results of the subgroup analysis, genotype-guided strategy significantly reduced the risk of MACE compared to STD group in both $<12$ months and $\geq 12$ months subgroups. Therefore, genotype-guided strategy might not only reduce the incidence of MACE in the early stage after PCI (0-6 months), during which adverse reactions were most likely to occur, but also improve the longterm efficacy outcome.

\section{Future research orientation}

Future relevant studies may need to focus more on the study population with stable CAD rather than ACS. Moreover, studies aiming at the de-escalation treatment instead of escalation treatment after genotyping could consummate the realization of genotype-guided antiplatelet therapy. The reduced risk of MACEs in Chinese population was found in the GENE group of our meta-analysis, and the same results were observed in several cohort studies based on the Chinese population $(43,44)$. Therefore, a relevant study in the non-Chinese population may be needed, especially in the American and African population.

\section{Limitations}

There were several limitations ineluctably in this meta-analysis. First, slight differences existed in the definitions of the MACEs, treatment strategies, and genotyping systems among included studies, which may affect the reliability of pooled RR. However, the sensitivity analysis and subgroup analysis we performed confirmed that these differences did not affect the final results. Second, most of the included studies were open-label without performing the blinding method, so that the selection biases were inevitable. However, low-risk bias was demonstrated in most aspects of the quality assessment, so the results of the meta-analysis should be significative. Third, some studies might not be included on account of the unavailable full-text.

\section{CONCLUSIONS}

The current meta-analysis results showed that genotype-guided antiplatelet treatment could reduce the risk of both composite and individual outcomes 
of MACEs without increasing the risk of bleeding events as compared with the standard treatment in patients with CAD or those undergoing PCI. However, this conclusion might be more applicable to escalation treatment strategy rather than deescalation treatment strategy. In addition, genotypeguided antiplatelet treatment might benefit Chinese population (or population with a high proportion of LOF allele carriers) or patients with ACS. In the context of the current increasing use of ticagrelor for patients after PCI, the effect of genotype-guided deescalation treatment needs to be further verified.

ACKNOWLEDGMENTS. Borui Tang and Xin Wang contributed equally. They drafted the original manuscript and contributed to the study selection, data extraction and data analysis. X.R.W contributed to the methodological support. Z.M and L.H.L conceived the study, resolved the disagreements and were responsible for the project supervision. All authors participated in the review \& editing of the final manuscript.

FUNDING. This manuscript has been produced without funding.

DATA AVAILABILITY. The data that support the findings of this study are available from the corresponding authors upon reasonable request.

CONFLICT OF INTEREST. The authors declare that they have no conflict of interest.

\section{REFERENCES}

1. Collet J-P, Thiele H, Barbato E, Barthélémy $\mathrm{O}$, Bauersachs J, Bhatt DL, et al. 2020 ESC Guidelines for the management of acute coronary syndromes in patients presenting without persistent STsegment elevation. Eur Heart J. 2020 doi:10.1093/eurheartj/ehaa575.

2. Valgimigli M, Bueno H, Byrne RA, Collet J-P, Costa F, Jeppsson A, et al. 2017 ESC focused update on dual antiplatelet therapy in coronary artery disease developed in collaboration with EACTS: The Task Force for dual antiplatelet therapy in coronary artery disease of the European Society of Cardiology (ESC) and of the European Association for Cardio-Thoracic Surgery (EACTS). Eur Heart J. 2018;39(3):213-60 doi:10.1093/eurheartj/ehx419.

3. Levine GN, Bates ER, Bittl JA, Brindis RG, Fihn SD, Fleisher LA, et al. 2016 ACC/AHA Guideline Focused Update on Duration of Dual Antiplatelet Therapy in Patients With Coronary Artery Disease:
A Report of the American College of Cardiology/American Heart Association Task Force on Clinical Practice Guidelines. J Am Coll Cardiol. 2016;68(10):1082-115 doi:10.1016/j.jacc.2016.03.513.

4. Matetzky S, Shenkman B, Guetta V, Shechter M, Beinart R, Bienart R, et al. Clopidogrel resistance is associated with increased risk of recurrent atherothrombotic events in patients with acute myocardial infarction. Circulation. 2004;109(25):3171-5

5. Corbett SJ, Ftouh S, Lewis S, Lovibond K. Acute coronary syndromes: summary of updated NICE guidance. $\quad$ BMJ. 2021;372:m4760 doi:10.1136/bmj.m4760.

6. Wallentin L, Becker RC, Budaj A, Cannon CP, Emanuelsson H, Held C, et al. Ticagrelor versus clopidogrel in patients with acute coronary syndromes. N Engl J Med. 2009;361(11):1045-57 doi:10.1056/NEJMoa0904327.

7. Wiviott SD, Braunwald $\mathrm{E}, \mathrm{McCabe} \mathrm{CH}$, Montalescot G, Ruzyllo W, Gottlieb S, et al. Prasugrel versus clopidogrel in patients with acute coronary syndromes. N Engl J Med. 2007;357(20):2001-15

8. Navarese EP, Khan SU, Kołodziejczak M, Kubica $\mathrm{J}$, Buccheri S, Cannon CP, et al. Comparative Efficacy and Safety of Oral P2Y(12) Inhibitors in Acute Coronary Syndrome: Network MetaAnalysis of 52816 Patients From 12 Randomized Trials. Circulation. 2020;142(2):150-60 doi:10.1161/circulationaha.120.046786.

9. Gimbel M, Qaderdan K, Willemsen L, Hermanides $\mathrm{R}$, Bergmeijer T, de Vrey E, et al. Clopidogrel versus ticagrelor or prasugrel in patients aged 70 years or older with non-ST-elevation acute coronary syndrome (POPular AGE): the randomised, open-label, non-inferiority trial. Lancet. 2020;395(10233):1374-81 doi:10.1016/S0140-6736(20)30325-1.

10. Storey RF, Bliden KP, Patil SB, Karunakaran A, Ecob R, Butler K, et al. Incidence of dyspnea and assessment of cardiac and pulmonary function in patients with stable coronary artery disease receiving ticagrelor, clopidogrel, or placebo in the ONSET/OFFSET study. J Am Coll Cardiol. 2010;56(3):185-93 doi:10.1016/j.jacc.2010.01.062.

11. Bonaca MP, Bhatt DL, Cohen M, Steg PG, Storey $\mathrm{RF}$, Jensen EC, et al. Long-term use of ticagrelor in patients with prior myocardial infarction. N Engl J Med.

2015;372(19):1791-800 doi:10.1056/NEJMoa1500857.

12. Simon T, Verstuyft C, Mary-Krause M, Quteineh L, Drouet E, Méneveau N, et al. Genetic determinants of response to clopidogrel and cardiovascular events. N Engl J Med. 2009;360(4):363-75 doi:10.1056/NEJMoa0808227.

13. Shuldiner AR, O'Connell JR, Bliden KP, Gandhi A, 
Ryan K, Horenstein RB, et al. Association of cytochrome P450 2C19 genotype with the antiplatelet effect and clinical efficacy of clopidogrel therapy. Jama. 2009;302(8):849-57 doi:10.1001/jama.2009.1232.

14. Mega JL, Simon T, Collet JP, Anderson JL, Antman EM, Bliden K, et al. Reduced-function CYP2C19 genotype and risk of adverse clinical outcomes among patients treated with clopidogrel predominantly for PCI: a meta-analysis. Jama. 2010;304(16):1821-30

doi:10.1001/jama.2010.1543.

15. Aradi D, Kirtane A, Bonello L, Gurbel PA, Tantry US, Huber K, et al. Bleeding and stent thrombosis on P2Y12-inhibitors: collaborative analysis on the role of platelet reactivity for risk stratification after percutaneous coronary intervention. European heart journal. 2015;36(27):1762-71 doi:10.1093/eurheartj/ehv104.

16. Winter MP, Schneeweiss T, Cremer R, Biesinger B, Hengstenberg C, Prüller F, et al. Platelet reactivity patterns in patients treated with dual antiplatelet therapy. European journal of clinical investigation. 2019;49(6):e13102 doi:10.1111/eci.13102.

17. Wallentin L, James S, Storey RF, Armstrong M, Barratt BJ, Horrow J, et al. Effect of CYP2C19 and ABCB1 single nucleotide polymorphisms on outcomes of treatment with ticagrelor versus clopidogrel for acute coronary syndromes: a genetic substudy of the PLATO trial. Lancet (London, England). 2010;376(9749):1320-8 doi:10.1016/s0140-6736(10)61274-3.

18. Martin J, Williams AK, Klein MD, Sriramoju VB, Madan S, Rossi JS, et al. Frequency and clinical outcomes of CYP2C19 genotype-guided escalation and de-escalation of antiplatelet therapy in a realworld clinical setting. Genetics in medicine : official journal of the American College of Medical Genetics. 2020;22(1):160-9 doi:10.1038/s41436019-0611-1.

19. Wang X, Wang S, Yang J, Yu X, Liu L. Genotypeguided antiplatelet therapy compared with standard therapy for patients with acute coronary syndromes or undergoing percutaneous coronary intervention: A systematic review and meta-analysis. Thromb Res. 2020;193:130-8 doi:10.1016/j.thromres.2020.06.002.

20. Lyu SQ, Yang YM, Zhu J, Wang J, Wu S, Zhang H, et al. The efficacy and safety of CYP2C19 genotype-guided antiplatelet therapy compared with conventional antiplatelet therapy in patients with acute coronary syndrome or undergoing percutaneous coronary intervention: A metaanalysis of randomized controlled trials. Platelets. 2020;31(8):971-80 doi:10.1080/09537104.2020.1780205.

21. Zhang H, Xiang Q, Liu Z, Mu G, Xie Q, Zhou S, et al. Genotype-guided antiplatelet treatment versus conventional therapy: A systematic review and meta-analysis. $\mathrm{Br} \mathrm{J}$ Clin Pharmacol. 2020 doi:10.1111/bcp.14637.

22. Pereira NL, Farkouh ME, So D, Lennon R, Geller $\mathrm{N}$, Mathew V, et al. Effect of Genotype-Guided Oral P2Y12 Inhibitor Selection vs Conventional Clopidogrel Therapy on Ischemic Outcomes After Percutaneous Coronary Intervention: The TAILOR-PCI Randomized Clinical Trial. Jama. 2020;324(8):761-71 doi:10.1001/jama.2020.12443.

23. Shi X, Zhang Y, Zhang Y, Zhang R, Lin B, Han J, et al. Personalized Antiplatelet Therapy Based on CYP2C19 Genotypes in Chinese ACS Patients Undergoing PCI: A Randomized Controlled Trial. FRONTIERS IN CARDIOVASCULAR MEDICINE. 2021;8 doi:10.3389/fcvm.2021.676954.

24. Zhang M, Wang J, Zhang Y, Zhang P, Chao Y, Gao $\mathrm{M}$, et al. Effects of individualized antiplatelet therapy, based on CYP2C19 genotyping, on platelet function in patients underwent percutaneous coronary intervention. Perfusion. 2020:267659120978584

doi: $10.1177 / 0267659120978584$.

25. Wells GA, Elliott J, Kelly S, Bai Z, Boucher M, Skidmore B, et al. CADTH Optimal Use Reports. Dual Antiplatelet Therapy Following Percutaneous Coronary Intervention: Clinical and Economic Impact of Standard Versus Extended Duration. Ottawa (ON) this project. Funding was received for educational activities. Dr. Welsh declared having received funding from the following companies within five years preceding this project: Astra Zeneca, Bayer, BMS/Pfizer, Boehringer Ingelheim. Funding was received for advisory work, as well as educational and research activities.: Canadian Agency for Drugs and Technologies in Health

26. Copyright (C) 2019 Canadian Agency for Drugs and Technologies in Health.; 2019

27. Mehran R, Rao SV, Bhatt DL, Gibson CM, Caixeta A, Eikelboom J, et al. Standardized bleeding definitions for cardiovascular clinical trials: a consensus report from the Bleeding Academic Research Consortium. Circulation. 2011;123(23):2736-47

doi:10.1161/CIRCULATIONAHA.110.009449.

28. Tuteja S, Glick H, Matthai W, Nachamkin I, Nathan A, Monono K, et al. Prospective CYP2C19 Genotyping to Guide Antiplatelet Therapy Following Percutaneous Coronary Intervention: A Pragmatic Randomized Clinical Trial. Circ Genom Precis Med. 2020;13(1):e002640 doi:10.1161/CIRCGEN.119.002640.

29. Claassens DMF, Vos GJA, Bergmeijer TO, Hermanides RS, van 't Hof AWJ, van der Harst P, et al. A Genotype-Guided Strategy for Oral P2Y12 Inhibitors in Primary PCI. N Engl $\mathrm{J}$ Med. 2019;381(17):1621-31 
doi:10.1056/NEJMoa1907096.

30. Notarangelo FM, Maglietta G, Bevilacqua P, Cereda M, Merlini PA, Villani GQ, et al. Pharmacogenomic Approach to Selecting Antiplatelet Therapy in Patients With Acute Coronary Syndromes: The PHARMCLO Trial. J Am Coll Cardiol. 2018;71(17):1869-77 doi:10.1016/j.jacc.2018.02.029.

31. Tomaniak M, Kołtowski Ł, Kochman J, Huczek Z, Rdzanek A, Pietrasik A, et al. Can prasugrel decrease the extent of periprocedural myocardial injury during elective percutaneous coronary intervention? Polish archives of internal medicine. 2017;127(11):730-40 doi:10.20452/pamw.4087.

32. Tam CC, Kwok J, Wong A, Yung A, Shea C, Kong $\mathrm{SL}$, et al. Genotyping-guided approach versus the conventional approach in selection of oral P2Y12 receptor blockers in Chinese patients suffering from acute coronary syndrome. J Int Med Res. 2017;45(1):134-46 doi:10.1177/0300060516677190.

33. Xie X, Ma YT, Yang YN, Li XM, Zheng YY, Ma X, et al. Personalized antiplatelet therapy according to CYP2C19 genotype after percutaneous coronary intervention: a randomized control trial. Int $\mathrm{J}$ Cardiol. $2013 ; 168(4): 3736-40$ doi:10.1016/j.ijcard.2013.06.014.

34. Roberts JD, Wells GA, Le May MR, Labinaz M, Glover C, Froeschl M, et al. Point-of-care genetic testing for personalisation of antiplatelet treatment (RAPID GENE): a prospective, randomised, proofof-concept trial. The Lancet. 2012;379(9827):1705-11 doi:10.1016/s01406736(12)60161-5.

35. Al-Rubaish AM, Al-Muhanna FA, Alshehri AM, Al-Mansori MA, Alali RA, Khalil RM, et al. Bedside testing of CYP2C19 vs. conventional clopidogrel treatment to guide antiplatelet therapy in ST-segment elevation myocardial infarction patients. International journal of cardiology. 2021 doi:10.1016/j.ijcard.2021.08.051.

36. Angiolillo DJ, Rollini F, Storey RF, Bhatt DL, James S, Schneider DJ, et al. International Expert Consensus on Switching Platelet P2Y ReceptorInhibiting Therapies. Circulation. 2017;136(20):1955-75

doi:10.1161/CIRCULATIONAHA.117.031164.

37. Sibbing D, Aradi D, Alexopoulos D, Ten Berg J, Bhatt DL, Bonello L, et al. Updated Expert Consensus Statement on Platelet Function and Genetic Testing for Guiding P2Y(12) Receptor Inhibitor Treatment in Percutaneous Coronary Intervention. JACC Cardiovascular interventions. 2019;12(16):1521-37 doi:10.1016/j.jcin.2019.03.034.

38. Tan JW, Chew DP, Abdul Kader MAS, Ako J, Bahl VK, Chan M, et al. 2020 Asian Pacific Society of Cardiology Consensus Recommendations on the
Use of P2Y(12) Receptor Antagonists in the AsiaPacific Region. European cardiology. 2021;16:e02 doi:10.15420/ecr.2020.40.

39. Kheiri B, Osman M, Abdalla A, Haykal T, Pandrangi PV, Chahine A, et al. CYP2C19 pharmacogenetics versus standard of care dosing for selecting antiplatelet therapy in patients with coronary artery disease: A meta-analysis of randomized clinical trials. Catheter Cardiovasc Interv. 2019;93(7):1246-52 doi:10.1002/ccd.27949.

40. Claassens DM, Sibbing D. De-Escalation of Antiplatelet Treatment in Patients with Myocardial Infarction Who Underwent Percutaneous Coronary Intervention: A Review of the Current Literature. J Clin Med. 2020;9(9) doi:10.3390/jcm9092983.

41. Zhou Y, Ingelman-Sundberg M, Lauschke VM. Worldwide Distribution of Cytochrome P450 Alleles: A Meta-analysis of Population-scale Sequencing Projects. Clin Pharmacol Ther. 2017;102(4):688-700 doi:10.1002/cpt.690.

42. Fricke-Galindo I, Céspedes-Garro C, RodriguesSoares F, Naranjo MEG, Delgado Á, de Andrés F, et al. Interethnic variation of CYP2C19 alleles, 'predicted' phenotypes and 'measured' metabolic phenotypes across world populations. Pharmacogenomics J. 2016;16(2):113-23 doi:10.1038/tpj.2015.70.

43. Scott SA, Sangkuhl K, Stein CM, Hulot JS, Mega JL, Roden DM, et al. Clinical Pharmacogenetics Implementation Consortium guidelines for CYP2C19 genotype and clopidogrel therapy: 2013 update. Clin Pharmacol Ther. 2013;94(3):317-23 doi:10.1038/clpt.2013.105.

44. Shen D-L, Wang B, Bai J, Han Q, Liu C, Huang X$\mathrm{H}$, et al. Clinical Value of CYP2C19 Genetic Testing for Guiding the Antiplatelet Therapy in a Chinese Population. J Cardiovasc Pharmacol. 2016;67(3):232-6 doi:10.1097/FJC.0000000000000337.

45. Li, M., Zhou L, Zhan F, Yin N. Based on CYP2C19 genotype of clopidogrel and ticagrelor treatment of acute coronary syndromes individualized drug analysis. Chin Pharm J. 2019;54(9):753-60 doi:10.11669 
